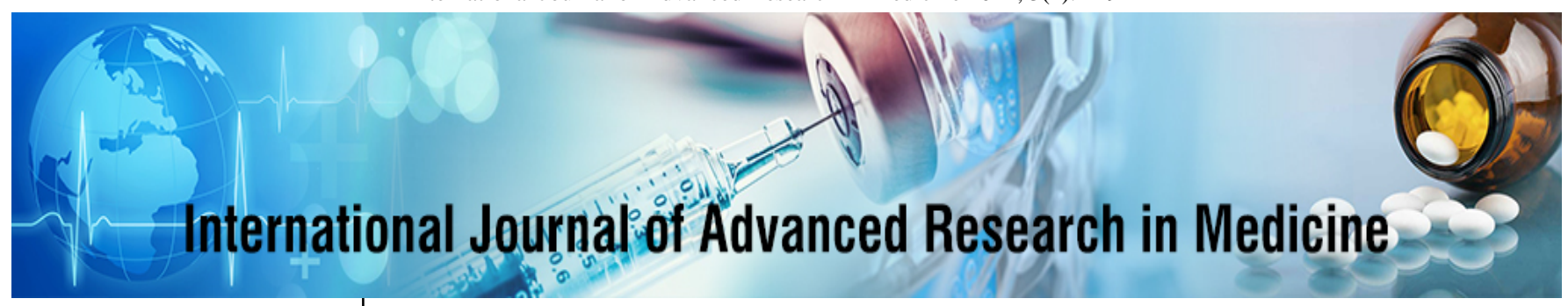

E-ISSN: 2706-9575

P-ISSN: 2706-9567

IJARM 2021; 3(2): 419-422

Received: 04-09-2021

Accepted: 27-10-2021

\section{Dr. Riji Varghese}

Assistant Professor, Department of General Medicine, Mount Zion Medical College, Chayalode, Adoor, Kerala, India

Dr. Shigil Mathew Varghese Assistant Professor, Department of General Medicine, S.A Medical College and Research

Foundation, Kozhencherry,

Pathanamthitta, Kerala, India
Corresponding Author: Dr. Shigil Mathew Varghese Assistant Professor, Department of General Medicine, S.A Medical College and Research

Foundation, Kozhencherry,

Pathanamthitta, Kerala, India

\title{
A study on relation between age and thyroid dysfunction in postmenopausal women
}

\section{Dr. Riji Varghese and Dr. Shigil Mathew Varghese}

DOI: https://doi.org/10.22271/27069567.2021.v3.i2g.283

\begin{abstract}
The balance of hormones in a woman's body changes during menopause. Lot of processes in the body are regulated by hormones, and it can take some time to adapt to the changes. The hormonal changes are sometimes, but not always, associated with menopause symptoms. Vasomotor symptoms affect up to $75 \%$ of peri-menopausal women. Project was submitted to ethical committee, got approval from the ethical committee. Test and control population were recruited after obtaining all necessary consent in their local regional language. As age increases, free T3 increases during age group (40-60) years, following which there is a dip in T3 values between $61-70$ years. But after the age of 70, free T3 again increases.
\end{abstract}

Keywords: age, thyroid dysfunction, postmenopausal women

\section{Introduction}

Menopause is an unspoken, unattended, reality of life, the cause of which is still undeciphered completely by man. Menopause, also known as the climacteric, is the time in most women's lives when menstrual periods stop permanently, and they are no longer able to have children ${ }^{[1]}$.

Menopause typically occurs between 45 and 55 years of age. Medical professionals often define menopause as having occurred when a woman has not had any vaginal bleeding for a year. It may also be defined by a decrease in hormone production by the ovaries. The balance of hormones in a woman's body changes during menopause. Lot of processes in the body are regulated by hormones, and it can take some time to adapt to the changes. The hormonal changes are sometimes, but not always, associated with menopause symptoms ${ }^{[2]}$. Vasomotor symptoms affect up to $75 \%$ of peri-menopausal women. Symptoms last for 12 years after menopause in most women, but may continue for up to 10 years or longer in others. Hot flushes are the primary reason women seek care at menopause. Hot flushes not only disturb women at work and interrupt daily activities, but also disrupt sleep. Many women report difficulty concentrating and emotional labiality during the menopausal transition ${ }^{[3]}$. Urogenital atrophy results in vaginal dryness and pruritus, dyspareunia, dysuria, and urinary urgency. Musculoskeletal symptoms characterized by backache, fractures on minimal trauma, decreased height, and mobility are common due to osteoporosis ${ }^{[4]}$. Although most women transition to menopause without experiencing psychiatric problems, an estimated $20 \%$ have depression at some point during menopause. Insomnia occurs in 40 $50 \%$ of women during the menopausal transition, and problems with sleep may or may not be connected to mood disorders ${ }^{[5-6]}$.

\section{Methodology}

- $\quad$ Project was submitted to ethical committee, got approval from the ethical committee. Test and control population were recruited after obtaining all necessary consent in their local regional language.

- $\quad$ The purpose of the study was explained to the patient and informed consent obtained.

- $\quad$ Patients were recruited as per inclusion- exclusion criteria.

- Relevant history including symptoms and signs at presentation, past medical history, drug history and examination findings noted. Data was collected using a proforma meeting the objectives of the study. 
In this method, the immobilization takes place at the surface of an opaque chemiluminescent reaction cell through the interaction of streptavidin coated on the opaque reaction cell and exogenously added biotinylated monoclonal antibody coupled to the analyte of interest. Upon mixing monoclonal biotinylated antibody, the enzyme labeled antibody and the test serum containing the native antigen, reaction results between the native antigen and the antibodies, without competition or stearic hindrance, to form a soluble sandwich complex. After equilibrium is attained, the antibody bound fraction is separated from unbound antigen by decantation or aspiration. The enzyme activity, determined by reaction with a substrate that generates light, in the antibody-bound fraction is directly proportional to the native antigen concentration. By utilizing several different serum reference of known antigen values, a dosage response curve can be generated from which the antigen concentration of an unknown analyte can be ascertained.

\section{Inclusion criteria}

- Postmenopausal women attending outpatient and inpatient of Medicine Department.

\section{Exclusion criteria}

- Known cases of diabetes mellitus

- Known cases of Thyroid dysfunction,

- Known cases of hypertension,

- Known cases of chronic kidney disease.

- $\quad$ Patients on Hormone replacement therapy

- Diagnosed cases of Ovarian and uterine malignancy

- Patients on drugs like iodide, amiodarone, salicylates, propranolol, octreotide, phenytoin, lithium, glucocorticoid, amphetamine, aminoglutethemide, somatostatins.

\section{Results}

Effect of age on thyroid profile was evaluated. Mean age for euthyroid subjects were 52.5 yrs, sub-clinical hypothyroid 53.6 yrs, overt hypothyroid 52.5 yrs and hyperthyroid subjects were 52.6 years. Results indicated that Free T4 and age have negative correlation. This means, as the age increases the Free T4 decreases and vice versa. Whereas $\mathrm{TSH}$ and age have positive correlation, that is, as the age increases the TSH also increases and vice versa. Similarly Free T3 and age also have positive correlation.

Table 1: Effect of age on thyroid profile

\begin{tabular}{|c|c|c|c|c|c|c|c|c|}
\hline & \multicolumn{2}{|c|}{ Euthyroid } & \multicolumn{2}{c|}{ Sub clinical hypothyroidism } & \multicolumn{2}{c|}{ Overt hypothyroidism } & \multicolumn{2}{c|}{ Hyperthyroidism } \\
\hline Variable & Mean & Std. Dev. & Mean & Std. Dev. & Mean & Std. Dev. & Mean & Std. Dev. \\
\hline N & 50 & & 28 & & 8 & & 14 & \\
\hline AGE & 52.5 & 12.1 & 53.6 & 10.3 & 52.5 & 12.4 & 52.6 & 10.5 \\
\hline
\end{tabular}

$\mathrm{P}$ Value $>0.05$

Table 1 shows the mean Age for Euthyroid (52.5 yrs), subclinical hypothyroid (53.6 yrs), overt hypothyroid (52.5 yrs) and hyperthyroidism (52.6 yrs). There is no statistically significant difference between the groups.

Table 2: Effect of age on thyroid profile

\begin{tabular}{|c|c|c|c|}
\hline Pearson correlation & Free t3 vs age & Free t4 vs age & Tsh vs age \\
\hline Correlation coefficient & 0.0285 & -0.0255 & 0.0986 \\
\hline P value & $>0.05$ & $>0.05$ & $>0.05$ \\
\hline
\end{tabular}

Table 2 shows, Free T4 and Age have negative correlation (means as the Age Increases the Free T4 Decreases and
Vice Versa), whereas TSH and Age have positive correlation (means as the age Increases the TSH also Increases and Vice Versa) similarly Free T3 and Age have positive correlation. All the three parameters Free T3, T4, TSH Vs Age are not statistically significant.

As age increases, free T3 increases during age group (4060) years, following which there is a dip in T3 values between $61-70$ years. But after the age of 70, free T3 again increases.

Free T3 increases as the age increases from fourths to sixties, following which there is a dip in free $\mathrm{t} 3$ values between $61-70$ yrs after which it increases as age increases

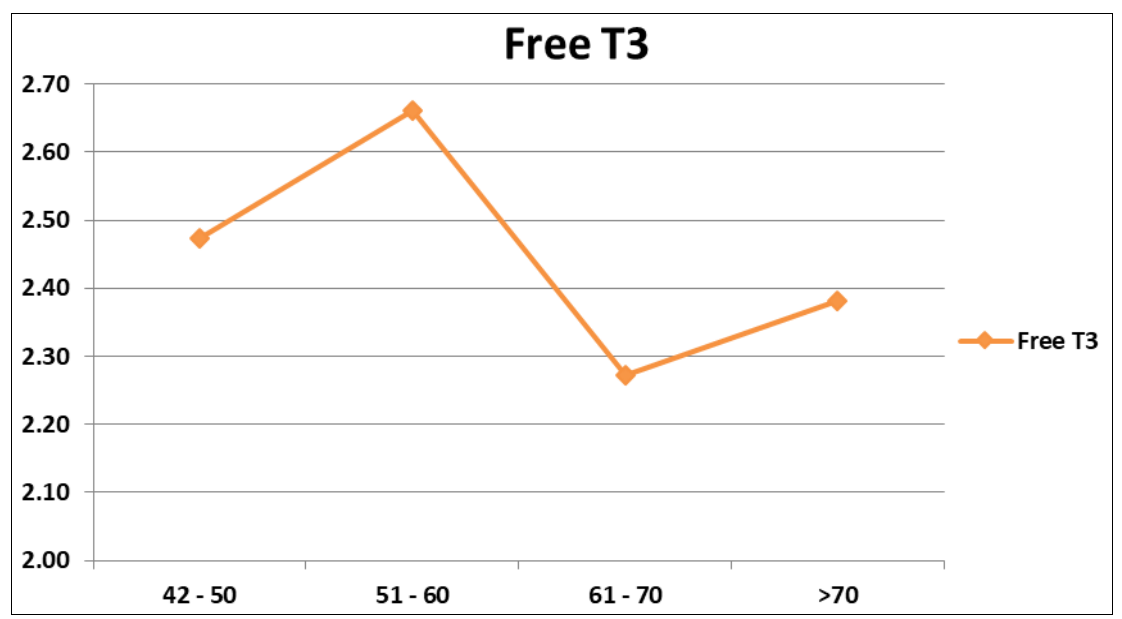

Graph 1: Age group and T3

As the age increases, Free T4 decreases in age group (4270) years. Following which freeT4 increases, as the age increases.
Free T4 decreases as the age increases from 42-70yrs, following which there is a increase in freeT4 as age increases. 


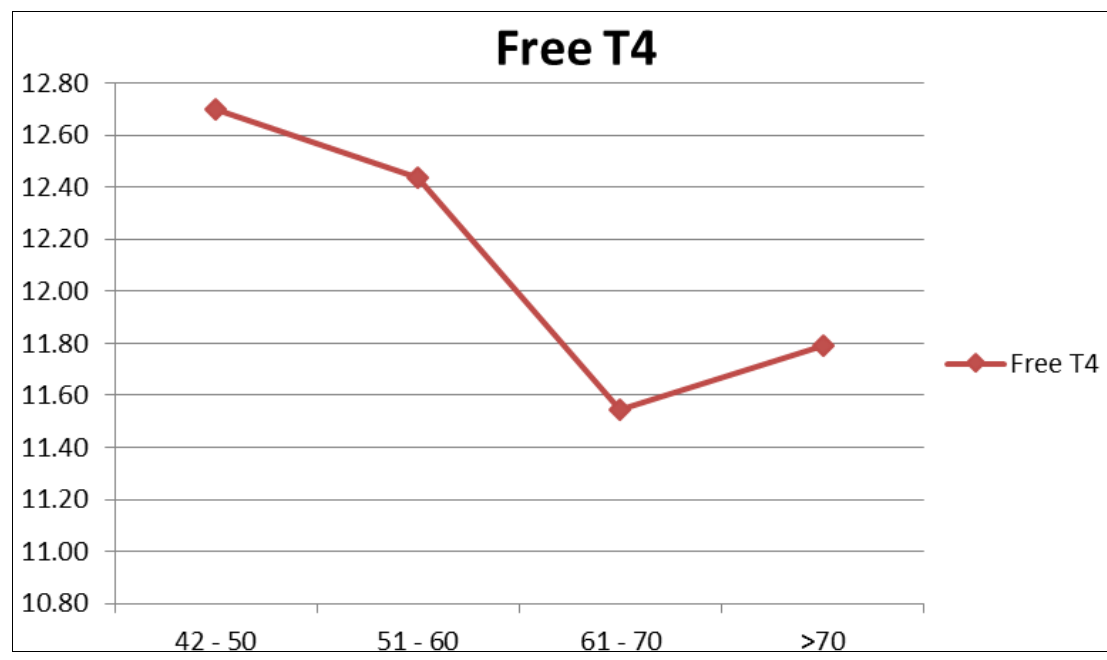

Graph 2: Age group and T4

This study shows that as the age increases, TSH increases in age group (42-70) years. But after 70 years, there is a dip in TSH values. TSH increases as the age increases from $42-$
70 yrs, following which there is a dip in TSH values after 70 yrs

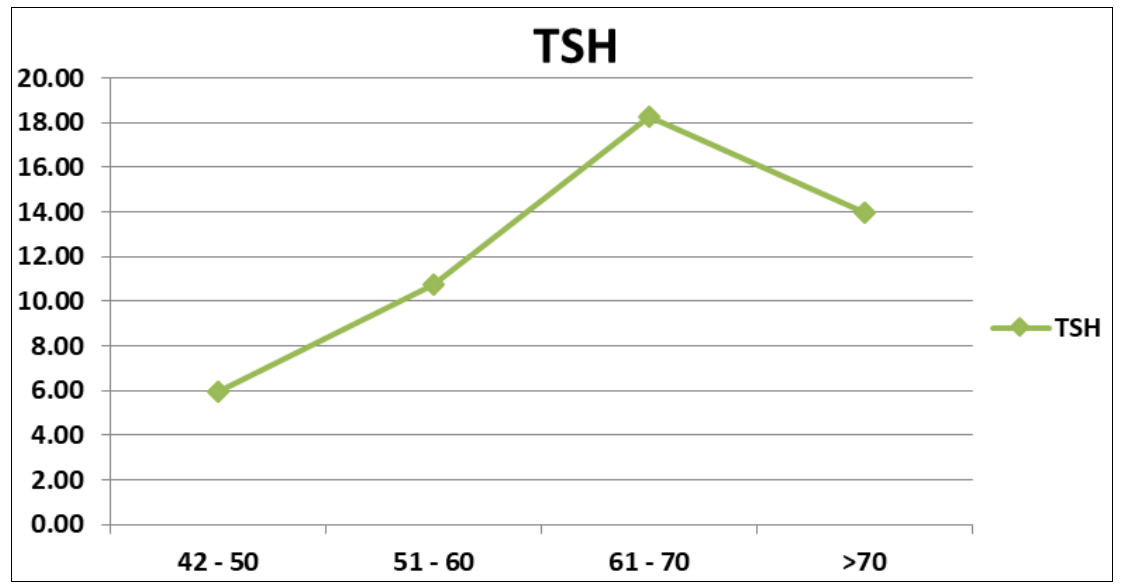

Graph 3: Age group and TSH

\section{Discussion}

Effect of age on thyroid profile was evaluated. Mean age for each group was calculated. Mean for euthyroid subjects were 52.5 yrs, sub-clinical hypothyroid 53.6 yrs, overt hypothyroid 52.5 yrs and hyperthyroid subjects were 52.6 yrs. Results indicated that Free T4 and age have negative correlation. Whereas TSH/T3 and age have positive correlation, that is, as the age increases the TSH/T3 also increases and vice versa. All the three parameters Free T3, T4, TSH with Age was not statistically significant.

Razvi S, Ingoe L, Keeka G et al,. [7] in 2007, In a randomized, double-blind, crossover study of L-thyroxine and placebo. One hundred patients with Subclinical hypothyroidism without previously treated thyroid or vascular disease were selected. 100 microg L-thyroxine or placebo daily for 12 wk each were given. Total cholesterol (TC) and endothelial function [brachial artery flowmediated dilatation], were assessed as an early marker of atherosclerosis. L-thyroxine treatment reduced TC (vs. placebo) from 231.6 to $220 \mathrm{mg} / \mathrm{dl}$, low-density lipoprotein cholesterol from 142.9 to $131.3 \mathrm{mg} / \mathrm{dl}$, waist to hip ratio from 0.83 to 0.81 ; and improved FMD from 4.2 to $5.9 \%, \mathrm{P}$ $<0.001$. Multivariate analysis showed that increased serum free $\mathrm{T}(4)$ level was the most significant variable predicting reduction in TC or improvement in flow mediated dilatation.
Furthermore, the symptom of tiredness improved on Lthyroxine therapy. SCH treated by L-thyroxine leads to a significant improvement in CV risk factors and symptoms of tiredness. The CV risk factor reduction is related to the increased level of achieved free T4 concentration.

Kitahara CM, Platz EA, Ladenson PW et al., ${ }^{[8]}$ in 2012, evaluated the association of central versus overall adiposity on levels of thyroid stimulating hormone (TSH), free triiodothyronine (fT(3)), and free thyroxine (fT(4)) among euthyroid subjects taken from a cross-sectional, representative sample of the adult non-institutionalized U.S. population. 1,623 men and 1,491 women who were 20 years and older, with no history of thyroid or liver disease, kidney failure, diabetes, or thyroid function-alteringmedication and having TSH, fT(3), and $\mathrm{fT}(4)$ levels between 0.5-4.49 $\mathrm{mIU} / \mathrm{L}, 2.5-3.9 \mathrm{pg} / \mathrm{mL}$, and 0.6-1.6 ng/dL were included. An increase in serum TSH levels was observed for every 1quartile increase in BMI in euthyroid men (3.8\% [95\% CI $0.8 \%, 6.8 \%]$ ) and euthyroid women (4.0\% [95\% CI 1.6\%, $6.5 \%]$ ). Results support that BMI and waist circumference are positively associated with levels of serum TSH and $f$ $\mathrm{T}(3)$ but not fT(4) among euthyroid adults.

Tognini S, Polini A, Pasqualetti G et al. ${ }^{[9]}$ in 2012, studied association between mild thyroid failure and lipid profile, primarily regarding serum triglyceride values and patients 
with slightly elevated thyrotropin (TSH, $<10 \mathrm{mIU} / \mathrm{L}$ ). In this study, the possible influence of gender and age on this relationship was assesed. The study included 2308 consecutive patients who were seen for suspected or diagnosed thyroid disease (1874 women, 434 men, mean age $47.5 \pm 14.1$ and $46.9 \pm 14.0$ years, respectively) and on whom studies of thyroid status and lipoprotein profile were conducted after an overnight fast. Patients with uncontrolled diabetes mellitus and those taking lipid-lowering drugs were excluded. There were 628 patients receiving L-thyroxine who had a diagnosis of hypothyroidism: 200 were hyperthyroid, and 120 were still hypothyroid. Overall, 648 patients were hypothyroid, and 290 were hyperthyroid. Total cholesterol (TC) and low-density lipoprotein cholesterol (LDLc) values as well as the LDL/high-density lipoprotein cholesterol (HDLc) ratio were elevated. This study confirms a gender differentiation in the relationship between hypothyroidism and the lipid profile, which is substantially influenced by age, especially in patients with mild thyroid impairment ${ }^{[10]}$.

\section{Conclusion}

As age increases, free T3 increases during age group (4060) years, following which there is a dip in T3 values between $61-70$ years. But after the age of 70 , free T3 again increases.

\section{References}

1. Sundaram V, Hanna AN, Koneru L, Newman HAI, M J Falko. "Both hypothyroidism and hyperthyroidism enhance low density lipoprotein oxidation,” Journal of Clinical Endocrinology and Metabolism 1997;82(10):3421-3424.

2. F Costantini S, Pierdomenico D, De Cesare D et al., "Effect of thyroid function on LDL oxidation," Arteriosclerosis, Thrombosis, and Vascular Biology 1998;18(5):732-737.

3. Diekman T, Demacker PNM, Kastelein JJP, Stalenhoef AFH, Wiersinga WM. "Increased oxidizability of lowdensity lipoproteins in hypothyroidism," Journal of Clinical Endocrinology and Metabolism 1998;83(5):1752-1755.

4. Torun AN, Kulaksizoglu S, Kulaksizoglu M, Pamuk BO, Isbilen E, Tutuncu NB. "Serum total antioxidant status and lipid peroxidation marker malondialdehyde levels in overt and subclinical hypothyroidism," Clinical Endocrinology 2009;70(3):469-474.

5. Kim SK, Kim SH, Park KS, Park SW, Cho YW. "Regression of the increased common carotid arteryintima media thickness in subclinical hypothyroidism after thyroid hormone replacement,” Endocrine Journal 2009;56(6):753-758.

6. Biondi B, Palmieri EA, Fazio S et al., "Endogenous subclinical hyperthyroidism affects quality of life and cardiac morphology and function in young and middleaged patients," Journal of Clinical Endocrinology and Metabolism 2000;85(12):4701-4705.

7. Razvi S, Ingoe L, Keeka G. The beneficial effect of LThyroxine on cardiovascular risk factors endothelial function in subclinical hypothyroidism. randomised crossover trial. Journal of Clinical Endocrinology and Metabolism 2007;92:1715-1723.

8. Kitahara CM. Body fatness and markers of thyroid function among U.S. men and women. PLoS One 2012;7(4):e34979.

9. Tognini S, Polini A, Pasqualetti G et al. Age and gender substantially influence the relationship between thyroid status and the lipoprotein profile: results from a large cross-sectional study. Thyroid 2012;22:10961103.

10. Reinehr $\mathrm{T}$, de Sousa G, Andler W. Hyperthyrotropinemia in obese is reversible after weight loss and is not related to lipids. J Clin Endocrinol Metab 2006;91:3088-3091. 